\title{
Dissociating proactive and reactive control in the Stroop task
}

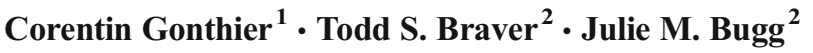

Published online: 9 February 2016

(C) Psychonomic Society, Inc. 2016

\begin{abstract}
The Dual Mechanisms of Control framework posits the existence of two distinct control mechanisms, proactive and reactive, which may operate independently. However, this independence has been difficult to study with most experimental paradigms. The Stroop task may provide a useful way of assessing the independence of control mechanisms because the task elicits two types of proportion congruency effects, list-wide and item-specific, thought to reflect proactive and reactive control respectively. The present research tested whether these two proportion congruency effects can be used to dissociate proactive and reactive control. In 2 separate participant samples, we demonstrate that list-wide and itemspecific proportion congruency effects are stable, exist in the same participants, and appear in different task conditions. Moreover, we identify two distinct behavioral signatures, the congruency cost and the transfer cost, which doubly dissociate the two effects. Together, the results are consistent with the view that proactive and reactive control reflect independent mechanisms.
\end{abstract}

Keywords Cognitive control · Dual Mechanisms of Control (DMC) - Stroop interference $\cdot$ List-wide proportion congruency $\cdot$ Item-specific proportion congruency

Corentin Gonthier

corentin.gonthier@gmail.com

1 Université Savoie Mont Blanc, LPNC UMR CNRS 5105, Chambéry, France

2 Department of Psychological and Brain Sciences, Washington University in Saint Louis, Campus Box 1125, One Brookings Dr., St. Louis, MO 63130, USA
Cognitive control, defined as the ability to regulate behavior according to a specific goal, holds a central place in human cognition but has proved difficult to decompose into component processes. The Dual Mechanisms of Control (DMC) framework posits two distinct control mechanisms (Braver, 2012; Braver, Gray, \& Burgess, 2007), proactive and reactive. The proactive mechanism exerts a form of preparatory control via sustained maintenance of goal-relevant information; this information is used to actively bias the processing of incoming information according to task goals. Reactive control, by contrast, functions as a late correction mechanism: task goals are transiently retrieved only after a demanding event has occurred. The two mechanisms are complementary and come with different costs and benefits, which implies that the optimal control mode may vary depending on the situation (Braver et al., 2007).

When variability in cognitive control exists, it is generally described in terms of a shift from one mode of control to the other (e.g., Barch et al., 2001; Braver, Paxton, Locke, \& Barch, 2009; Paxton, Barch, Racine, \& Braver, 2008). This suggests the possibility that the two mechanisms constitute the two poles of a single dimension, with participants applying the same cognitive control process at different points in time - either before or after the critical event occurs. By contrast, theoretical descriptions of the DMC framework (Braver, 2012; Braver et al., 2007) raise the idea that proactive and reactive control could actually constitute independent mechanisms, but this idea has not been directly investigated. Importantly, a critical evaluation of the independence of proactive and reactive control requires an appropriate experimental paradigm, in which the two mechanisms can be selectively manipulated in different experimental conditions and in which they elicit different response patterns. In the current study, we draw upon recent findings suggesting that the Stroop task represents just such a paradigm, and we use this task to assess the independence of proactive and reactive control. 


\section{Independent control effects in the Stroop task}

The Stroop task (Stroop, 1935), which requires naming the ink color of color name words, produces interference on incongruent trials in which the word name and ink color do not match (e.g., the word RED printed in green ink). Cognitive control is necessary in the Stroop task to overcome interference on incongruent trials; for example, one classic account suggests that reading the word is the dominant response in the task, and that cognitive control is required to selectively orient attention and processing toward the naturally weaker response of naming the color (Miller \& Cohen, 2001). Critically, cognitive control demands in the Stroop task can be manipulated by varying the proportion congruency ( $\mathrm{PC}$; that is, the proportion of congruent items) in the task (Logan \& Zbrodoff, 1979; see also Tzelgov, Henik, \& Berger, 1992). In mostly congruent (high PC) conditions, cognitive control is seldom required since reading the words leads to a correct response on most trials; in contrast, in mostly incongruent (low PC) conditions, cognitive control is consistently needed to overcome the interference encountered on most trials.

Prior studies have demonstrated that PC manipulations can be implemented in a list-wide (LWPC) or item-specific (ISPC) manner, and LWPC and ISPC effects have been interpreted as reflecting different mechanisms (for reviews, see Bugg, 2012; Bugg \& Crump, 2012). The LWPC effect refers to the robust finding that Stroop interference is significantly reduced when most of the trials in a task block are incongruent, as compared with a mostly congruent block (e.g. Bugg, 2014a; Bugg \& Chanani, 2011; Bugg, McDaniel, Scullin, \& Braver 2011; Hutchison, 2011; Kane \& Engle, 2003; Lindsay \& Jacoby, 1994; Logan \& Zbrodoff, 1979; Lowe \& Mitterer, 1982). The classic interpretation of this effect is that participants who encounter a high number of incongruent trials in a task block implement a strategic or global form of control to bias their attention away from the irrelevant dimension of word reading throughout the task block (Lindsay \& Jacoby, 1994; Logan \& Zbrodoff, 1979; Lowe \& Mitterer, 1982; for alternative views, see Blais \& Bunge, 2010; Bugg, Jacoby, $\&$ Toth, 2008; Schmidt, 2013, 2014). The ISPC effect is conceptually similar, except that PC is manipulated at the level of items within a task block; interference is reduced for mostly incongruent items as opposed to mostly congruent ones (Bugg \& Hutchison, 2013; Bugg, Jacoby, \& Chanani, 2011; Bugg et al., 2008; Hutchison, 2011; Jacoby, Lindsay, \& Hessels, 2003). The ISPC effect has been attributed to an item-level control mechanism, selectively engaged upon presentation of an item — or a feature of an item - associated with high levels of interference (i.e., with conflict), signaling high control demands (Bugg, 2014b; Bugg \& Hutchison, 2013; Bugg, Jacoby, et al., 2011; Shedden, Milliken, Watter, \& Monteiro, 2013; but see Schmidt \& Besner, 2008). Based on these conceptual distinctions, the LWPC and ISPC effects have been hypothesized to reflect the operation of proactive and reactive control mechanisms, respectively (Bugg, 2012; Hutchison, 2011).

Experimental findings have provided evidence that LWPC and ISPC effects reflect distinct mechanisms: ISPC effects are observed in the absence of LWPC effects (e.g., Bugg, Jacoby, et al., 2011), whereas LWPC effects have been observed for items matched in item-specific proportion congruency (Bugg, 2014a; Bugg \& Chanani, 2011; Bugg, Diede, Cohen-Shikora, \& Selmeczy, 2015; Bugg, McDaniel, et al., 2011); moreover, pure LWPC effects tend to disappear with cognitive aging (Bugg, 2014a), while ISPC effects are found in both young and older adults (Bugg, 2014b). Importantly, however, no prior study to date has directly contrasted independent indices of LWPC and ISPC effects in the same participants. Some studies have observed only one of the two effects (Blais \& Bunge, 2010; Bugg 2014a, Experiments 1b and 2a; Bugg et al., 2008), others have used designs where one of the two PC effects was explicitly neutralized (Bugg \& Chanani, 2011; Bugg, Jacoby, et al., 2011), and others have used designs where the ISPC effect could not be assessed independently of the LWPC effect because ISPC was manipulated within a mostly congruent or mostly incongruent task block (Bugg, 2014a, Experiments 1a and 2b; Bugg \& Chanani, 2011; Hutchison, 2011).

In summary, proactive and reactive control are hypothesized to constitute independent mechanisms, but this hypothesis has not been directly tested; in parallel, LWPC and ISPC effects observed in the Stroop task are thought to constitute dissociable indices of proactive and reactive control, but independent estimates of these effects have never been assessed in the same participants. The goal of the present research was to take advantage of LWPC and ISPC effects to doubly dissociate proactive and reactive control by directly comparing their behavioral signatures. Observing different signatures for the two mechanisms would provide strong evidence that they constitute different processes, rather than the two ends of a continuum.

\section{Rationale for the present study}

In order to contrast the behavioral signatures of proactive and reactive control, the current study was designed to obtain independent estimates of ISPC and LWPC effects; indeed, ISPC and LWPC effects are generally confounded in experimental studies (see Blais \& Bunge, 2010; Bugg et al., 2008), which makes it difficult to disentangle their separate contributions to performance. To this end, the two effects were manipulated independently, and they were tested in separate task blocks. To study the ISPC effect, participants completed an item-specific (IS) task block including an equal number of items with $25 \%$ and $75 \%$ proportion congruency (PC-25 [mostly incongruent] and PC-75 [mostly congruent] items, respectively); the listwide PC was $50 \%$ in this task block, so as to avoid any 
contribution of the LWPC effect. The item-by-item variation in control demands and the unbiased list-wide PC were expected to elicit reactive control. To study the LWPC effect, participants completed both a list-wide mostly congruent (LWmc) and a list-wide mostly incongruent (LWmi) task block; control demands were consistently high in the LWmi block and consistently low in the LWmc block. In contrast with the item-by-item variation in control demands in the IS block, the consistent presence of high interference in the LWmi block was hypothesized to favor a global control mechanism. As a consequence, we expected the LWmi condition to engage proactive control when compared to the LWmc condition. Additionally, all three task blocks (LWmc, LWmi, IS) included a separate set of unbiased items with $50 \%$ proportion congruency (PC-50), making it possible to examine LWPC effects independently of item-specific influences. A key component of the present study was the use of a withinsubjects design, wherein all participants completed all task blocks. Such a design was necessary to provide evidence that reactive and proactive control are dissociable in the same participants, and to appropriately contrast their behavioral signatures.

Based on the theoretical functioning of proactive and reactive control, we predicted that the two mechanisms would be associated with the same benefit but with unique costs. In particular, we took as the marker of improved control the standard measure that has been utilized in the literature: the magnitude of the Stroop interference effect (i.e., incongruent congruent reaction time). We predicted that both the list-wide and item-specific manipulations of proportion congruency (i.e., mostly incongruent vs. mostly congruent) would lead to a reduction of the Stroop interference effect (i.e., a benefit of enhanced cognitive control). Critically, this Stroop interference benefit was predicted to be similar in magnitude for proactive and reactive control mechanisms. This common benefit makes it challenging to dissociate the two mechanisms. However, one important prediction is that the benefit of proactive control and the benefit of reactive control should not be positively correlated if the LWPC and ISPC effects are indeed caused by independent mechanisms.

The more critical prediction is that although the two mechanisms should produce similar control benefits, they should also be associated with distinct costs due to engaging a particular mode of cognitive control. In particular, engagement of proactive control was expected to be associated with a congruency cost - a relative slowing on congruent trials associated with mostly incongruent items compared to mostly congruent items. The rationale was based on the well-established finding that participants tend to respond faster on congruent trials, as they partly rely on word reading (i.e., the facilitation effect; MacLeod, 1991). Because engagement of proactive control tends to bias participants away from word reading in advance of stimulus onset (e.g., Lindsay \& Jacoby, 1994), the congruency cost would be observed in terms of a reduction in the facilitation found on congruent trials. Importantly, this cost was not expected to accompany the reactive control mode, because reactive control is thought to be implemented in a stimulus-triggered manner, and only for items associated with high levels of interference (i.e., incongruent trials; see Blais, Robidoux, Risko, \& Besner, 2007; Verguts \& Notebaert, 2008).

Conversely, however, reactive control but not proactive control was predicted to be associated with a transfer cost, meaning that the benefit of reactive control should not transfer to the unbiased (i.e., $50 \%$ congruent) items with lower control demands. In particular, we predicted that in the item-specific block the magnitude of the Stroop effect for unbiased items would be significantly greater than the magnitude of the Stroop effect observed for the high cognitive control, mostly incongruent items. This transfer cost was expected to selectively appear for reactive control because reactive control is selectively implemented for items with high control demands (i.e., incongruent trials for the mostly incongruent items), whereas the global nature of proactive control means that its influence should transfer to all items within a task block, including unbiased items. In other words, we predicted a smaller transfer cost in the LWmi block associated with proactive control. ${ }^{1}$ The pattern of predicted results is summarized in Fig. 1.

A key concern of the present study was the stability of the findings: the indices used to dissociate LWPC and ISPC effects had unknown psychometric properties, and several of these indices were based on difference scores, which tend to demonstrate low reliability (Thomas \& Zumbo, 2012). To ensure that the effects of interest were stable across samples, the study was based on two different subexperiments, which were close replications of each other. All participants in Experiment 1a were Washington University in Saint Louis undergraduates, whereas Experiment $1 \mathrm{~b}$ involved a larger and more diverse community sample. The materials and procedure for the two experiments were identical, except that the order of experimental conditions was changed from Experiment 1a to Experiment $1 \mathrm{~b}$ to ensure that the results were not due to an order effect.

\footnotetext{
${ }^{1}$ It is important to note the conceptual distinction between a marker of cognitive control and a cost of cognitive control. As described previously, we consider the reduction of Stroop interference effects observed in both the list-wide and item-specific mostly incongruent conditions (relative to mostly congruent) to be the positive consequence of engaged cognitive control. In contrast, the congruency cost and transfer costs are negative consequences, and thus undesirable (but likely) by-products of engaging in these distinct forms of control. Thus, only the Stroop effect magnitude should be considered a marker of the strength of cognitive control engagement.
} 
(a)

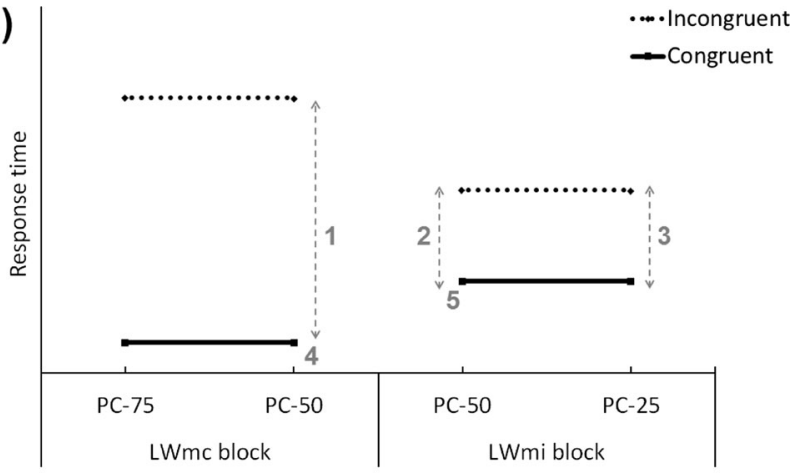

(b)

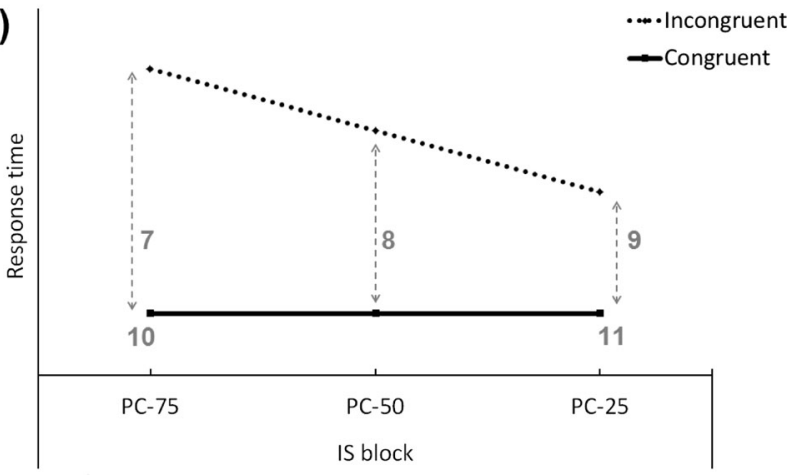

Fig. 1 Hypothesized pattern of results for proactive control (LWPC; top panel) and reactive control (ISPC; bottom panel). (a) Proactive control was expected to decrease Stroop interference from the LWmc block to the LWmi block even for unbiased items (arrow $1>$ arrow 2), to elicit no transfer cost from biased to unbiased items (arrow $2=$ arrow 3 ), and to elicit a congruency cost in the form of a slowing in response times on congruent trials (point $5>$ point 4). (b) Analogously, reactive control was expected to decrease Stroop interference from PC-75 to PC-25 items (arrow $7>$ arrow 9), but to also elicit a transfer cost from biased to unbiased items (arrow $8>$ arrow 9), and to elicit no congruency cost (point $10=$ point 11 ). The two mechanisms were expected to decrease Stroop interference by a similar amount (arrow $1-$ arrow 2 = arrow $7-$ arrow 9)

\section{Method $^{2}$}

\section{Participants}

All participants completed the study for payment (\$10.00 an hour). All participants were native English speakers and had normal or corrected-to-normal vision; none of them had completed the picture--word Stroop task before. All participants provided informed consent prior to the experiment. Participants in Experiment 1a were 35 undergraduates at Washington University in Saint Louis (12 males, mean age $=20.9$ years).

\footnotetext{
${ }^{2}$ Relevant material for this paper can be accessed via Open Science Framework at osf.io/b9zyv/. This repository contains the task scripts, the raw data, and a summary document detailing all of the analyses conducted for the manuscript, using the R statistical language. This document should provide the code needed to reproduce the relevant results contained within the manuscript. Please note that this material has not been peer-reviewed.
}

Participants in Experiment 1b were 58 individuals (15 males, mean age $=26.0$ years) recruited from the community through a participant registry; the mean education level was college graduate, and 21 of the participants were college students.

\section{Design and stimuli}

All participants completed two experimental conditions. The first condition was designed to test the LWPC effect; it comprised the LWmc task block, which included PC-75 items, and the LWmi task block, which included PC-25 items. The second condition was designed to test the ISPC effect; it comprised the IS task block, which included both PC-75 and PC-25 items. In other words, in the LW condition, the manipulation of proportion congruency (LWmi vs. LWmc) was implemented in a list-wide fashion (i.e., between blocks), whereas in the IS condition the analogous manipulation (PC-75 vs. PC-25) was implemented in a trial-by-trial fashion (i.e., within a block). A small number of unbiased PC-50 items appeared in each task block to allow for unbiased assessment of transfer benefits. For each item type within each task block, trials could be either congruent or incongruent.

In the classic color-word Stroop paradigm, the ISPC effect is sometimes attributed to the formation of simple stimulusresponse associations rather than to an actual cognitive control mechanism (Bugg \& Hutchison, 2013; Bugg, Jacoby, et al., 2011; Schmidt \& Besner, 2008); to avoid this potential problem, participants completed a version of the picture-word Stroop task designed to orient participants toward relying on item-specific control rather than associative learning mechanisms (for details, see Bugg, Jacoby, et al., 2011, Experiment 2). Eight black-and-white drawings of animals were used as stimuli (see Bugg, Jacoby, et al., 2011, for a detailed description of the stimuli). The pictures were divided into two sets. The first set comprised four animals (frog, cow, pig, seal) that were used for the unbiased PC-50 items in all three task blocks (LWmc, LWmi, and IS). The second set comprised four animals (cat, dog, bird, fish) that were used for the biased items, but with varying proportion congruencies across the LWmc and LWmi blocks (i.e., these items all had PC-75 in the LWmc block and PC-25 in the LWmi block). In the IS task block, where there were intermixed PC-75 and PC-25 items, two animal pictures in the set (e.g. bird, cat) were used for PC-75 items and the other two animal pictures (e.g. dog, fish) were used for PC-25 items (counterbalanced across participants).

A word corresponding to an animal name was superimposed on each drawing. The word matched the drawing in congruent, but not in incongruent trials (see Fig. 2 for an example). In incongruent trials, the word was the name of one of the other three animals from the same set of items; in other words, a picture of a bird could be accompanied by the words fish, dog or cat, but not by the word frog, whereas a picture of a frog could not be accompanied by the word bird. 

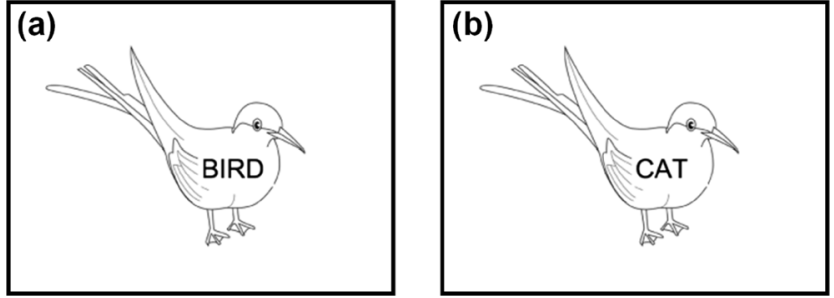

Fig. 2 Illustration of sample congruent (a) and incongruent (b) stimuli used in the Stroop task

\section{Procedure}

All participants were tested individually and received the instruction to name the animals in the pictures as quickly and as accurately as possible. The participant's responses were timed with a voice key (E-prime SRBOX device; Schneider, Eschman, \& Zuccolotto, 2002). Stimuli were displayed at the center of a 17-inch screen, and remained on screen until the voice-key detected an answer. An experimenter was present throughout the testing session and manually coded the participant's answers on a keyboard. The experimenter coded a scratch trial when the participant provided an unclear answer, or when the voice-key was not tripped by the onset of the participant's answer. Trials were separated by a $1,000 \mathrm{~ms}$ interstimulus interval, which started as soon as the experimenter coded the participant's answer.

Participants performed 384 trials in the LWmc task block (96 PC-50 trials and 288 PC-75 trials), 384 trials in the LWmi task block ( 96 PC-50 trials and 288 PC-25 trials), and 432 trials in the IS task block (192 PC-75 trials, 192 PC-5 trials, and 48 PC-50 trials appearing only in the second half of the block $^{3}$ ). The arrangement of stimuli is summarized in Table 1 . Participants received a short break halfway through each task block. Before each task block, participants performed a series of 22 practice trials with the same list-wide and item-specific proportion congruencies as the following experimental trials.

Experiment la was broken down into two sessions performed at most 1 week apart. All participants performed the LWmc task block in a first session and the IS and LWmi task blocks in a second session; each task block lasted approximately 20 minutes. The procedure of Experiment $1 \mathrm{~b}$ was identical to Experiment 1a with one exception: the experiment was completed in a single session lasting approximately 1 hour. To ensure that the results of Experiment 1a were not due to an order effect, the order of task blocks was changed in Experiment $1 \mathrm{~b}$ : participants completed the LWmc block, the LWmi block and the IS block, in order.

\footnotetext{
${ }^{3}$ This design and the number of trials per trial type were chosen for consistency with the two prior studies that observed a pure LWPC effect (Bugg \& Chanani, 2011) and a pure ISPC effect (Bugg, Jacoby, et al., 2011) using the picture-word Stroop. Specifically, the study observing an ISPC effect (Bugg, Jacoby, et al., 2011) used more biased trials and restricted the presentation of unbiased trials to the final task block.
}

Table 1 Example arrangement of stimuli as a function of task block

\begin{tabular}{|c|c|c|c|c|c|c|}
\hline \multirow[t]{2}{*}{ Task block } & \multirow[t]{2}{*}{ Item type } & \multirow[t]{2}{*}{ Picture } & \multicolumn{4}{|l|}{ Word } \\
\hline & & & CAT & DOG & FISH & BIRD \\
\hline \multirow[t]{9}{*}{ LWmc block } & \multirow[t]{5}{*}{ PC-75 } & Cat & 54 & 6 & 6 & 6 \\
\hline & & Dog & 6 & 54 & 6 & 6 \\
\hline & & Fish & 6 & 6 & 54 & 6 \\
\hline & & Bird & 6 & 6 & 6 & 54 \\
\hline & & & COW & FROG & PIG & SEAL \\
\hline & \multirow[t]{4}{*}{ PC-50 } & Cow & 12 & 4 & 4 & 4 \\
\hline & & Frog & 4 & 12 & 4 & 4 \\
\hline & & Pig & 4 & 4 & 12 & 4 \\
\hline & & Seal & 4 & 4 & 4 & 12 \\
\hline \multirow[t]{10}{*}{ LWmi block } & & & CAT & DOG & FISH & BIRD \\
\hline & \multirow[t]{5}{*}{ PC-25 } & Cat & 18 & 18 & 18 & 18 \\
\hline & & Dog & 18 & 18 & 18 & 18 \\
\hline & & Fish & 18 & 18 & 18 & 18 \\
\hline & & Bird & 18 & 18 & 18 & 18 \\
\hline & & & COW & FROG & PIG & SEAL \\
\hline & \multirow[t]{4}{*}{ PC-50 } & Cow & 12 & 4 & 4 & 4 \\
\hline & & Frog & 4 & 12 & 4 & 4 \\
\hline & & Pig & 4 & 4 & 12 & 4 \\
\hline & & Seal & 4 & 4 & 4 & 12 \\
\hline \multirow[t]{10}{*}{ IS block } & & & CAT & DOG & FISH & BIRD \\
\hline & \multirow[t]{2}{*}{ PC-75 } & Cat & 72 & 8 & 8 & 8 \\
\hline & & Dog & 8 & 72 & 8 & 8 \\
\hline & \multirow[t]{3}{*}{ PC-25 } & Fish & 24 & 24 & 24 & 24 \\
\hline & & Bird & 24 & 24 & 24 & 24 \\
\hline & & & COW & FROG & PIG & SEAL \\
\hline & \multirow[t]{4}{*}{ PC-50 } & Cow & 6 & 2 & 2 & 2 \\
\hline & & Frog & 2 & 6 & 2 & 2 \\
\hline & & Pig & 2 & 2 & 6 & 2 \\
\hline & & Seal & 2 & 2 & 2 & 6 \\
\hline
\end{tabular}

\section{Results}

Accuracy and average RTs were computed for each experimental condition, with average RTs computed on correct trials only. All trials with RTs lower than 200 ms or higher than 3, $000 \mathrm{~ms}$ were dropped from the analysis; this included less than $1 \%$ of trials in all conditions. Participants with a Stroop effect more than three standard deviations from the mean in a condition were also excluded from further analyses; four participants were excluded for this reason (one participant in Experiment 1 and three participants in Experiment 2).

A series of preliminary analyses was performed to confirm the invariance of the effects across the two experiments. These analyses were computed by testing the interaction between the effect of interest and the participant sample, treated as a categorical variable (i.e., Experiment 1a vs. Experiment 1b); a significant interaction would indicate that the effect of interest 
varied as a function of the sample. The results revealed that none of the effects of interest varied across samples, all $p$ s $>$ .10; as a consequence, the data for the two subexperiments were combined for all subsequent analyses. The total sample size comprised 89 participants. Descriptive statistics for all trial types are presented in Table 2 . The results are presented with the magnitude of the Stroop effect (i.e., incongruent congruent) as the dependent measure unless otherwise noted. The complete results of ANOVAs using trial type as an additional factor are reported in Table 3.

\section{Evidence of independent LWPC and ISPC effects}

The first step of the analysis was to provide evidence for the existence of LWPC and ISPC effects in independent conditions; the objective was to replicate, in the current withinsubjects design, the patterns previously observed in separate studies. We first examined the prediction that Stroop interference would be reduced for the LWmi list compared to the LWmc list due to the implementation of proactive control. When considering biased (PC-25 and PC-75) items, the LWPC manipulation successfully modulated the Stroop effect, with reduced Stroop interference in the LWmi task block $(M=63 \mathrm{~ms})$ compared to the LWmc task block $(M=111 \mathrm{~ms}), t(88)=11.67, p$ $<.001, \eta_{p}^{2}=.61$. The error rate data supported this conclusion (LWmi task block: $M=.016$; LWmc task block: $M=.040), t(88)=7.63, p<.001, \eta_{p}^{2}=.40$. More importantly, a similar effect was observed for the unbiased (PC-50) items that were matched across lists, with reduced Stroop interference in the LWmi task block $(M=86 \mathrm{~ms})$ compared to the LWmc task block $(M=115 \mathrm{~ms}), t(88)=6.42, p<.001, \eta_{p}^{2}=.43$ (see Fig. 3). Again, the error rate data supported this conclusion (LWmi task block: $M=.012$; LWmc task block: $M=.025), t(88)=3.17, p=.002, \eta_{p}^{2}=.10$.

The second analysis tested the independent existence of an ISPC effect by examining biased items in the IS task block; we expected interference to be reduced for PC-25 items compared to PC-75 items due to the implementation of reactive control. For response times, the ISPC manipulation successfully modulated the Stroop effect, with reduced Stroop interference for PC-25 items $(M=64 \mathrm{~ms})$ compared to PC-75 items $(M=88 \mathrm{~ms}), t(88)=5.78$, $p<.001, \eta_{p}^{2}=.28$ (see Fig. 4). The error rate data again supported this conclusion, with reduced Stroop interference for PC-25 items $(M=.016)$ compared to PC-75 items $(M=.030), t(88)=3.71, p<.001, \eta_{p}^{2}=.14$. In summary, both the LWPC and the ISPC manipulations were successful in independently reducing the magnitude of the Stroop effect, thus extending prior literature (e.g. Bugg, 2014a; Bugg \& Chanani, 2011; Bugg, Jacoby, et al., 2011; Bugg, McDaniel, et al., 2011).

\section{Comparing the behavioral signatures of LWPC and ISPC effects}

The primary goal of the study was to directly contrast select behavioral signatures across the LWPC and ISPC conditions, in order to provide evidence for dissociable patterns of proactive and reactive control, respectively. ${ }^{4}$ First, we examined the benefit of cognitive control by assessing the reduction in Stroop interference from the mostly congruent to the mostly incongruent condition. For the LWPC effect, this was achieved by comparing PC-50 items in the $\mathrm{LWmc}$ and LWmi task blocks; for the ISPC effect, we compared PC-75 and PC-25 items in the IS task block, as in the previous series of analyses. A 2 (PC effect: LWPC, ISPC) $\times 2$ (condition: mostly congruent, mostly incongruent) ANOVA conducted on RTs did not reveal a two-way interaction, $F(1,88)=$ $0.63, M S E=1023, p=.430, \eta_{p}^{2}=.01$, indicating that the reduction in Stroop interference from the mostly congruent to the mostly incongruent condition was similar for the LWPC effect $(M=29 \mathrm{~ms})$ and for the ISPC effect $(M=24$ $\mathrm{ms})$. In other words, the two control mechanisms elicited the same benefit in reducing Stroop interference to a comparable extent.

If these two effects were generated by the same control mechanism, a positive correlation between them would be expected. Instead, we observed a significant, but weak, negative correlation between the two benefit indices, $r(87)=-.26$, $p=.015$. In other words, participants who demonstrated a larger reduction in interference due to the LWPC effect tended to demonstrate a slightly smaller reduction in interference due to the ISPC effect. ${ }^{5}$ This correlation is represented in Fig. 5. The presence of a negative relationship between the two indices is consistent with the DMC framework, given that individuals predominantly biased to utilize proactive control would have less of a demand to engage reactive control. However, it is not consistent with the idea of a single control mechanism generating both effects.

Second, we tested the hypothesis that the congruency cost would be greater for proactive than for reactive control. This hypothesis was tested by examining whether the reduction in

\footnotetext{
${ }^{4}$ There were 288 biased trials of each type and 96 PC-50 trials in the two LW task blocks, when compared to 192 biased trials of each type and 48 PC-50 trials in the IS block; this may be a cause for concern when directly comparing effects across blocks. Importantly, however, all the analyses yielded similar results when comparing only the first or the last 192 biased trials and 48 PC-50 trials in the LW blocks to the IS block. In other words, the differences in behavioral signatures cannot be explained by differences in the absolute number of trials.

${ }^{5}$ Anecdotally, the two benefit indices also bore different relationships to average response times: participants who demonstrated a large reduction in interference due to the LWPC effect had faster overall response times, $r(88)=-.32, p=.002$, whereas the reduction in interference due to the ISPC effect was completely unrelated to response times, $r(88)=-.01, p=$ .918 . These results provide preliminary evidence for divergent validity of the two indices.
} 
Table 2 Descriptive statistics for response times and error rates as a function of task block, item-specific PC, and type of trial

Item-specific proportion congruency Task block

\begin{tabular}{|c|c|c|c|c|c|c|c|c|c|}
\hline & \multirow{2}{*}{\multicolumn{3}{|c|}{$\overline{\mathrm{LWmc}}$}} & & & & & & \\
\hline & & & & \multicolumn{3}{|l|}{ LWmi } & \multicolumn{3}{|l|}{ IS } \\
\hline & $\mathrm{CON}$ & INC & Stroop & $\mathrm{CON}$ & INC & Stroop & $\mathrm{CON}$ & INC & Stroop \\
\hline \multicolumn{10}{|c|}{ Response times } \\
\hline PC-75 & $625(77)$ & $736(87)$ & $111(42)$ & & & & $642(95)$ & $730(106)$ & $88(40)$ \\
\hline PC-50 & $680(84)$ & 795 (91) & $115(49)$ & $698(93)$ & 784 (113) & $86(48)$ & $687(100)$ & 787 (118) & $100(55)$ \\
\hline PC-25 & & & & $660(97)$ & $723(101)$ & $63(30)$ & $635(96)$ & $699(102)$ & $64(29)$ \\
\hline \multicolumn{10}{|l|}{ Error rates } \\
\hline PC-75 & $.002(.004)$ & $.042(.039)$ & $.040(.039)$ & & & & .005 (.007) & $.035(.033)$ & $.030(.035)$ \\
\hline PC-50 & $.008(.012)$ & $.033(.056)$ & $.025(.055)$ & $.011(.020)$ & $.021(.038)$ & $.012(.039)$ & $.009(.024)$ & $.028(.035)$ & $.019(.040)$ \\
\hline PC-25 & & & & $.004(.008)$ & $.020(.020)$ & $.016(.022)$ & $.003(.008)$ & $.019(.019)$ & $.016(.019)$ \\
\hline
\end{tabular}

Note. Average values with standard deviations in parentheses. CON congruent trials, INC incongruent trials, Stroop magnitude of the Stroop effect computed as incongruent - congruent

proportion congruency from the mostly congruent to the mostly incongruent condition produced significant slowing for RTs on congruent trials, and critically, whether this slowing differed in the LWPC and ISPC conditions. Again, the analysis compared PC-50 items in the LWmc and LWmi condition for the LWPC effect, and the PC-75 and PC-25 items in the IS block for the ISPC effect. A 2 (PC effect: LWPC, ISPC) $\times 2$ (condition: mostly congruent, mostly incongruent) ANOVA restricted to congruent trials revealed a significant two-way interaction, $F(1,88)=18.13, \operatorname{MSE}=741, p<.001, \eta_{p}^{2}=$ .17 , indicating that the congruency cost was only present in the LW blocks $(M=17 \mathrm{~ms})$ and not in the IS block $(M=-7$ $\mathrm{ms})$. In other words, only proactive control elicited a congruency cost.

Third, we tested the hypothesis that the transfer cost would be greater for reactive than for proactive control. The transfer cost should be reflected in significantly greater Stroop interference on the unbiased PC-50 items relative to the mostly incongruent PC-25 items. More specifically, proactive control should elicit a smaller transfer cost than reactive control due to its global nature, which means the increase in Stroop interference from the mostly incongruent PC-25 items to the unbiased PC-50 items should be smaller in the LWmi block than in the IS block. A 2 (task block: LWmi, IS) $\times 2$ (item type: PC-25, PC-50) ANOVA conducted on RTs revealed a significant twoway interaction, $F(1,88)=5.60, M S E=763, p=.020, \eta_{p}^{2}=$ .06 , suggesting that the increase in interference from PC-25 to PC-50 items differed as a function of task block. This two-way interaction was then decomposed into main effects. For the LWmi block, the main effect of item type was significant, $t(88)=5.40, p<.001, \eta_{p}^{2}=.25$, indicating a significant increase in interference from PC-25 to PC-50 items ( $M=22$ $\mathrm{ms})$; the effect of item type was also significant for the IS block, $t(88)=6.78, p<.001, \eta_{p}^{2}=.34$, but the increase in interference from PC-25 to PC-50 items was about 50\% larger
( $M=36 \mathrm{~ms}$ ), as was the corresponding effect size. In other words, the analysis indicated a higher transfer cost for reactive control.

Overall, the results indicated that the pattern of costs and benefits was different for proactive and reactive control: the benefit of control, namely the reduction of Stroop interference associated with low proportion congruency, was similar for the two mechanisms; however, proactive control elicited a higher congruency cost whereas reactive control elicited a higher transfer cost, as predicted. These findings are summarized in Fig. 6. A final analysis was performed to test whether the interaction between control mechanism and type of cost was significant. A 2 (control mechanism: proactive, reactive) $\times 2$ (type of cost: congruency cost, transfer cost) ANOVA indicated no main effect of control mechanism, $F(1,88)=$ $1.90, M S E=1338.7, p=.171, \eta_{p}^{2}=.02$, suggesting that no particular mechanism was associated with higher costs in general. However, the cross-over two-way interaction between mechanism and type of cost was significant, $F(1,88)=$ $19.68, M S E=1669.5, p<.001, \eta_{p}^{2}=.18$, indicating that the pattern of costs doubly dissociated proactive and reactive control, as predicted.

\section{General discussion}

The objective of this research was to provide evidence for the dissociation of proactive and reactive control by eliciting separable LWPC and ISPC effects, and by contrasting their behavioral signatures in a within-subjects design. Consistent with our hypotheses, the results indicated that both LWPC and ISPC effects emerged in the same participants. This finding constitutes an extension of the prior studies that observed one effect while controlling the other (e.g., Bugg \& Chanani, 2011; Bugg, Jacoby, et al., 2011) or that observed the two 
Table 3 Results for the omnibus ANOVAs as a function of condition

\begin{tabular}{|c|c|c|c|c|c|c|c|}
\hline & Factor (levels) & Dependent variable & $d f$ & $F$ & $M S E$ & $\eta_{p}^{2}$ & Significance \\
\hline \multirow{6}{*}{ LWPC effect for biased items } & \multirow[t]{2}{*}{ TT (2) } & RT & 1,88 & 683.49 & 987 & .89 & $* * *$ \\
\hline & & Error rate & 1,88 & 88.64 & .0008 & .50 & $* *$ \\
\hline & \multirow[t]{2}{*}{$\mathrm{PC}(2)$} & RT & 1,88 & 3.78 & 2856 & .04 & $\circ$ \\
\hline & & Error rate & 1,88 & 33.82 & .0002 & .28 & $* * *$ \\
\hline & \multirow[t]{2}{*}{$\mathrm{TT} * \mathrm{PC}$} & RT & 1,88 & 136.14 & 372 & .61 & $* * *$ \\
\hline & & Error rate & 1,88 & 58.25 & .0002 & .40 & $* * *$ \\
\hline \multirow[t]{6}{*}{ LWPC effect for PC-50 items } & \multirow[t]{2}{*}{ TT (2) } & RT & 1,88 & 476.54 & 1879 & .84 & $* * *$ \\
\hline & & Error rate & 1,88 & 15.91 & .002 & .15 & $* * *$ \\
\hline & \multirow[t]{2}{*}{$\mathrm{PC}(2)$} & RT & 1,88 & 0.24 & 2671 & .00 & \\
\hline & & Error rate & 1,88 & 2.04 & .0005 & .02 & \\
\hline & \multirow[t]{2}{*}{$\mathrm{TT} * \mathrm{PC}$} & RT & 1,88 & 41.27 & 465 & .32 & $* * *$ \\
\hline & & Error rate & 1,88 & 10.02 & .0004 & .10 & $* *$ \\
\hline \multirow[t]{6}{*}{ ISPC effect } & \multirow[t]{2}{*}{ TT (2) } & $\mathrm{RT}$ & 1,88 & 609.56 & 833 & .87 & $* * *$ \\
\hline & & Error rate & 1,88 & 96.83 & .0005 & .52 & $* * *$ \\
\hline & \multirow[t]{2}{*}{ PC (2) } & $\mathrm{RT}$ & 1,88 & 61.86 & 530 & .41 & $* * *$ \\
\hline & & Error rate & 1,88 & 25.95 & .0003 & .23 & $* * *$ \\
\hline & \multirow[t]{2}{*}{$\mathrm{TT} * \mathrm{PC}$} & RT & 1,88 & 33.42 & 383 & .28 & $* * *$ \\
\hline & & Error rate & 1,88 & 13.79 & .0003 & .14 & $* * *$ \\
\hline
\end{tabular}

Note. TT $=$ trial type ${ }^{\circ} p<.10 . * p<.05 . * * p<.01 . * * * p<.001$.

effects in different participants (Bugg, 2014a; Hutchison, 2011). In the present experiments, the two effects were fully dissociable, in that the LWPC effect appeared for unbiased (i.e., 50\% congruent) items whereas the ISPC effect appeared within an unbiased task block (with 50\% list-wide PC). These features suggest that the two effects do reflect the functioning of distinct control mechanisms. More importantly, proactive and reactive control were associated with different behavioral signatures: while both control mechanisms elicited a similar reduction in interference, proactive control was associated with a larger slowing in response times on congruent trials, whereas the reduction of interference associated with reactive

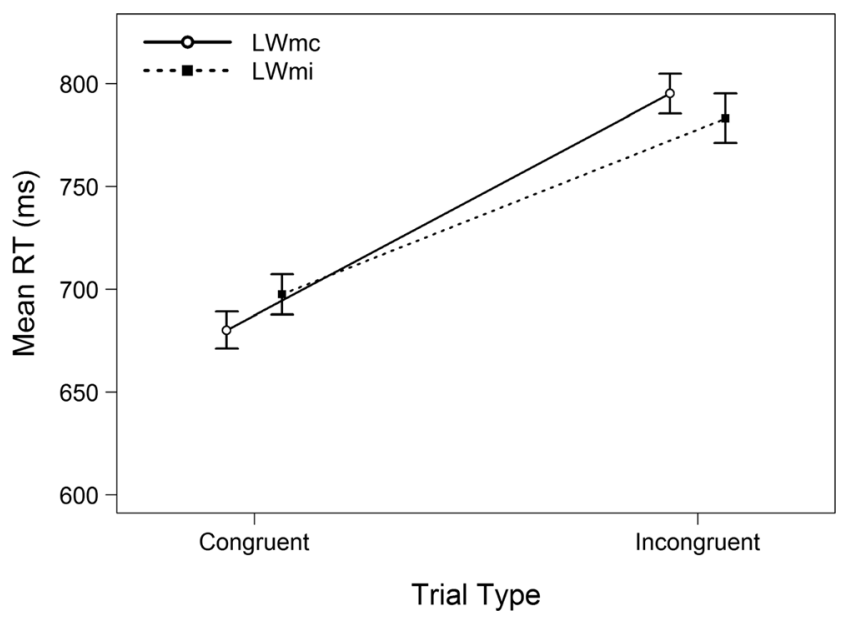

Fig. 3 Mean response time on PC-50 items as a function of task block and trial type. Error bars represent standard errors of the mean control demonstrated a smaller transfer to unbiased items. These different patterns strongly support the idea that proactive and reactive control constitute distinct and independent mechanisms, rather than a single mechanism acting at different points in time.

The specific behavioral signatures that we identifiedhigher congruency cost with the LWPC effect and higher transfer cost with the ISPC effect-were entirely compatible with the theoretical mechanisms of proactive and reactive control (Braver, 2012; Braver et al., 2007). Specifically, as proactive control would result in a global, sustained attentional bias toward color naming, it should both reduce the Stroop effect

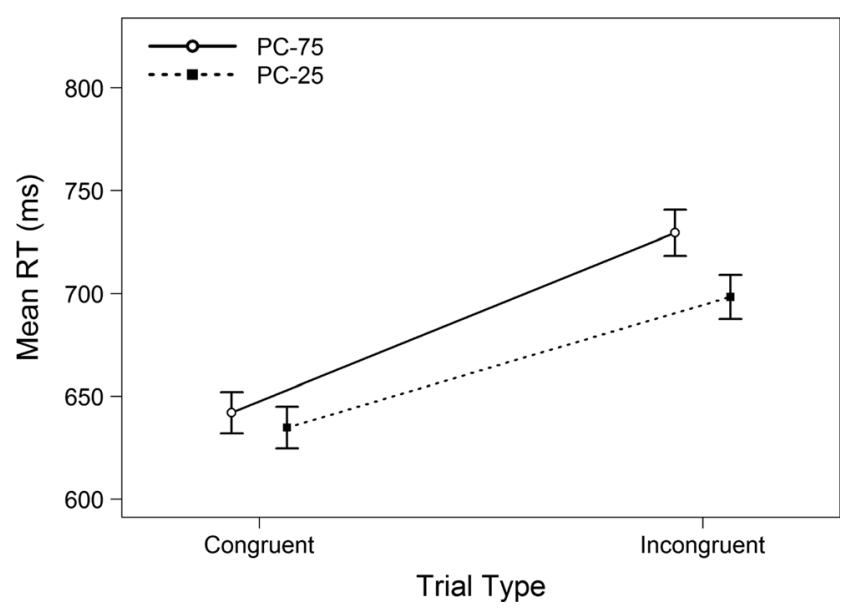

Fig. 4 Mean response time in the IS block as a function of item-specific proportion congruency and trial type. Error bars represent standard errors of the mean 


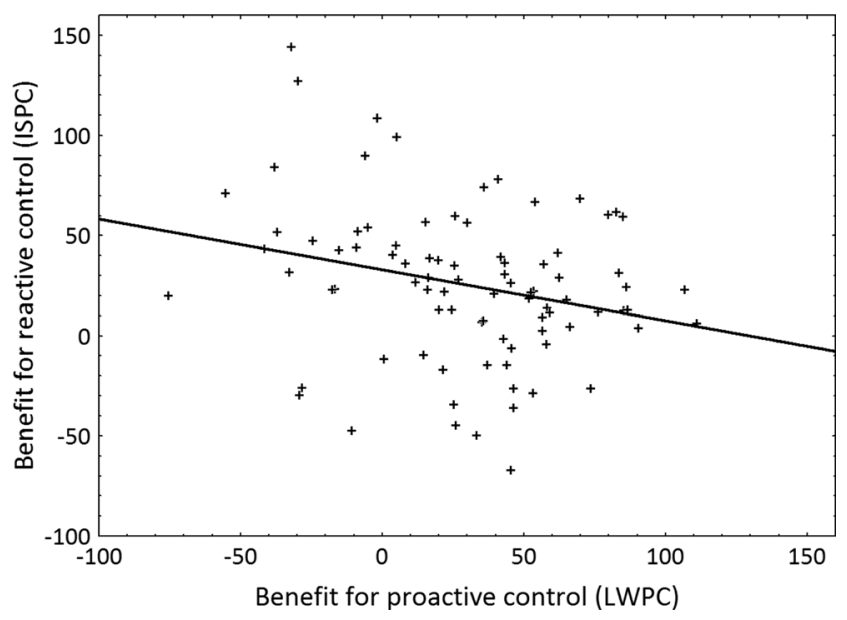

Fig. 5 Correlation between the benefit for using proactive control and the benefit for using reactive control

even on unbiased items (producing only a small transfer cost) and reduce the processing of word information even on congruent trials (producing a large congruency cost). Conversely, as reactive control should be triggered in an item-specific manner and only following the detection of interference, it should impact only biased items (resulting in a larger transfer

(a)

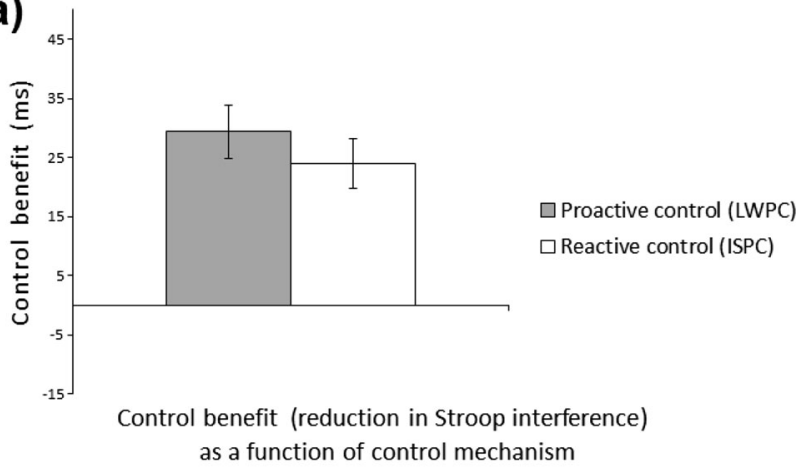

(b)

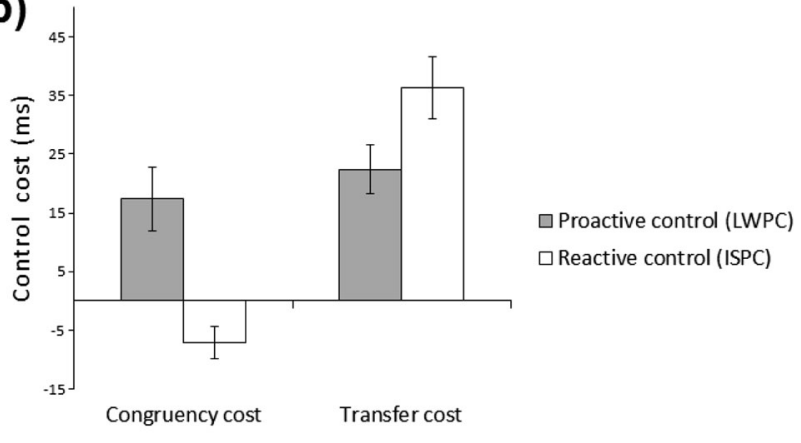

Control cost as a function of cost type and control mechanism

Fig. 6 Stroop interference benefit (a) and costs (b) as a function of control mechanism. Error bars represent standard errors of the mean. Control benefit $=$ reduction in Stroop interference from the mostly congruent to the mostly incongruent condition; congruency cost $=$ increase in RT on congruent trials from mostly congruent to mostly incongruent items; transfer cost $=$ increase in Stroop interference from mostly incongruent to unbiased items cost for unbiased items) and should not impact congruent trials (resulting in no congruency cost). The presence of these two behavioral signatures thus strongly supports the idea that LWPC and ISPC effects independently reflect proactive and reactive control, respectively. Importantly, the observed behavioral signatures were highly comparable across two different experiments, which suggests that the effects are relatively stable. These results therefore inform both the Stroop literature by firmly establishing the independence of LWPC and ISPC effects, and the cognitive control literature by providing a heuristically valuable paradigm from which to independently assess proactive and reactive control: the separability of the two effects suggests that they may be used to measure the efficiency of either control mechanism. For example, a high value of the LWPC effect in a given participant could indicate the operation of a very effective proactive control mechanism. Critically, the same participant could also demonstrate a high value of the ISPC effect, additionally reflecting a very effective reactive control mechanism.

Although our results suggest that proactive and reactive control constitute independent mechanisms, it is entirely possible that they do interact in normal situations. For example, a high level of proactive control in a task could make reactive control irrelevant, and thus less likely to be implemented by participants (Hutchison, Bugg, Lim, Olsen, in press). This idea is in line with the fact that cognitive control is costly in terms of cognitive resources (e.g., Braver et al., 2007); an individual would be unlikely to implement more control than required in a task. Interestingly, the idea that the two control mechanisms interact in normal situations could explain why variation in cognitive control generally appears as a shift from one mechanism to the other (e.g., Braver et al., 2009; Paxton et al., 2008): a decrease in the use of one mechanism could be offset by an increase in the use of the other, and vice versa. Indeed, the current results are also consistent with this idea, in that the reduction in Stroop interference associated with reactive control was negatively correlated with the reduction associated with proactive control. This finding can be interpreted in the same manner, that individuals more likely to utilize proactive control were faced with less of a demand to engage reactive control.

One limitation of the present work is that the number of trials was not balanced across all trial types: indeed, the unbiased PC-50 trials occurred less frequently than biased trials. Unexpectedly, the lower frequency of unbiased items was associated with overall slower response times for these items, independently of the task block and trial type (see Table 2); this relative slowing could introduce some noise in the results, all the more so since the relative frequency of unbiased trials was not constant as a function of task block. This limitation does not affect the analysis of control benefits and congruency costs, which did not rely on unbiased trials, but it could affect the analysis of transfer costs. The present design was dictated by strong practical constraints: only two prior studies have 
observed pure LWPC and ISPC effects using the picture-word Stroop task, and the number of trials was chosen for consistency with these works (Bugg \& Chanani, 2011; Bugg, Jacoby, et al., 2011). Moreover, it is difficult to equate the frequency of the different trial types without disrupting the LWPC; for example, increasing the number of PC-50 trials in a mostly congruent list would make the list less congruent overall. Nonetheless, future experiments should strive to improve the experimental paradigm by balancing the frequency of the different trial types as much as possible. In the present case, the disproportionate slowing on PC-50 trials is likely due to our use of a picture-word paradigm where the high perceptual dissimilarity between stimuli is likely to elicit a certain amount of surprise in participants when infrequent items are presented. Indeed, in studies in which the classic color-word Stroop paradigm is used, there is typically little slowing on infrequent unbiased items (Bugg, 2014a). ${ }^{6}$

The present work suggests several new lines of research. For example, there are strong conceptual reasons to map LWPC and ISPC effects onto proactive and reactive control mechanisms; however, more data is needed to ensure that PC effects are actually isomorphic with the two mechanisms of the DMC framework. Since the neural substrate of proactive and reactive control has been extensively studied, a possible solution would be to compare the neural signatures of LWPC and ISPC effects with other tasks involving proactive and reactive control. A close look at the descriptive statistics reveals another possible extension for the present results. It seemed to be the case that the magnitude of the Stroop effect was lower for PC-50 items than for PC-75 items in the LWmc task block, and participants also appeared to make fewer errors on PC-25 items than on PC-50 items in the LWmi task block; both differences were significant $(p \mathrm{~s}<.05)$. Likewise, when considering PC-50 items, the magnitude of Stroop interference was also lower for the LWmi task block (with 25\% list-wide PC) than for the IS task block (with 50\% list-wide PC), and lower for the IS task block than for the LWmc task block (with 75\% list-wide PC); again, both differences were significant $(p s<.05)$. These two observations suggest that an item-specific control mechanism may be operating within the LWmc and LWmi task blocks and that a global control mechanism may be operating across all three task blocks, including

\footnotetext{
$\overline{6}$ To test this assumption within the context of our present hypotheses, we conducted two follow-up studies $(N=50$ and $N=36)$ using the colorword Stroop but with a very similar within-subjects design examining both list-wide and item-specific proportion congruency manipulations. The proportion of PC-50 trials was also balanced across lists. In both studies, an almost identical pattern of primary results was obtained, replicating both the common Stroop interference benefits and dissociable costs of the two manipulations. However, and most critically, in these color-word studies we did not observe any appreciable slowing on PC50 items. These findings support the hypothesis that slowing observed in the picture-word Stroop data presented here might be due to the peculiarities of high perceptual dissimilarity among items.
}

the IS block. In other words, both the LWPC and ISPC effects seem to vary linearly along orthogonal dimensions, congruent with the idea that the two effects are truly doubly dissociated. Testing this idea thoroughly was not possible in the current design because PC-50 items were much less frequent than biased items, but providing more direct evidence for this hypothesis (e.g., comparing the magnitude of the ISPC effect at different levels of list-wide PC; cf. Hutchison, 2011) would provide stronger arguments in favor of the separability of proactive and reactive control.

\section{Conclusion}

List-wide proportion congruency and item-specific proportion congruency effects in the Stroop task are dissociable: they can be observed independently in different experimental conditions and they are associated with different behavioral signatures. These two proportion congruency effects may thus constitute independent reflections of proactive and reactive control mechanisms, and they may be used to separately assess the effectiveness of each mode of control.

Acknowledgments This research was supported by a grant from the National Institute of Mental Health (R37 MH066078). We thank Bridgette Shamleffer for assistance with data collection and analysis. We also wish to thank the reviewers, whose comments greatly improved the first version of the manuscript.

\section{References}

Barch, D. M., Carter, C. S., Braver, T. S., Sabb, F. W., MacDonald, A., Noll, D. C., \& Cohen, J. D. (2001). Selective deficits in prefrontal cortex function in medication-naive patients with schizophrenia. Archives of General Psychiatry, 58(3), 280-288. doi:10.1001/ archpsyc.58.3.280

Blais, C., \& Bunge, S. (2010). Behavioral and neural evidence for itemspecific performance monitoring. Journal of Cognitive Neuroscience, 22, 2758-2767. doi:10.1162/jocn.2009.21365

Blais, C., Robidoux, S., Risko, E. F., \& Besner, D. (2007). Item-specific adaptation and the conflict-monitoring hypothesis: A computational model. Psychological Review, 114(4), 1076-1086. doi:10.1037/ 0033-295X.114.4.1076

Braver, T. S. (2012). The variable nature of cognitive control: A dual mechanisms framework. Trends in Cognitive Sciences, 16(2), 106113. doi:10.1016/j.tics.2011.12.010

Braver, T. S., Gray, J. R., \& Burgess, G. C. (2007). Explaining the many varieties of working memory variation: Dual mechanisms of cognitive control. In A. R. A. Conway, C. Jarrold, M. J. Kane, A. Miyake, \& J. N. Towse (Eds.), Variation in working memory (pp. 76-106). New York, NY: Oxford University Press.

Braver, T. S., Paxton, J. L., Locke, H. S., \& Barch, D. M. (2009). Flexible neural mechanisms of cognitive control within human prefrontal cortex. PNAS Proceedings of the National Academy of Sciences of the United States of America, 106(18), 7351-7356. doi:10.1073/ pnas.0808187106 
Bugg, J. M. (2012). Dissociating levels of cognitive control: The case of Stroop interference. Current Directions in Psychological Science, 21(5), 302-309. doi:10.1177/0963721412453586

Bugg, J. M. (2014a). Conflict-triggered top-down control: Default mode, last resort, or no such thing? Journal of Experimental Psychology: Learning, Memory, and Cognition, 40(2), 567-587. doi:10.1037/ a0035032

Bugg, J. M. (2014b). Evidence for the sparing of reactive cognitive control with age. Psychology and Aging, 29(1), 115-127. doi:10.1037/ a0035270

Bugg, J. M., \& Chanani, S. (2011). List-wide control is not entirely elusive: Evidence from picture-word Stroop. Psychonomic Bulletin \& Review, 18, 930-936.

Bugg, J. M., \& Crump, M. J. C. (2012). In support of a distinction between voluntary and stimulus-driven control: A review of the literature on proportion congruent effects. Frontiers in Psychology, 3, 367. doi:10.3389/fpsyg.2012.00367

Bugg, J. M., Diede, N. T., Cohen-Shikora, E. R., \& Selmeczy, D. (2015). Expectations and experience: Dissociable bases for cognitive control? Journal of Experimental Psychology: Learning, Memory, and Cognition, 41, 1349-1373.

Bugg, J. M., \& Hutchison, K. A. (2013). Converging evidence for control of color-word Stroop interference at the item level. Journal of Experimental Psychology: Human Perception and Performance, 39(2), 433-449. doi:10.1037/a0029145

Bugg, J. M., Jacoby, L. L., \& Chanani, S. (2011). Why it is too early to lose control in accounts of item-specific proportion congruency effects. Journal of Experimental Psychology: Human Perception And Performance, 37(3), 844-859. doi:10.1037/a0019957

Bugg, J. M., Jacoby, L. L., \& Toth, J. P. (2008). Multiple levels of control in the Stroop task. Memory \& Cognition, 36(8), 1484-1494. doi:10. 3758/MC.36.8.1484

Bugg, J. M., McDaniel, M. A., Scullin, M. K., \& Braver, T. S. (2011). Revealing list-level control in the Stroop task by uncovering its benefits and a cost. Journal of Experimental Psychology: Human Perception And Performance, 37(5), 1595-1606. doi:10.1037/ a0024670

Hutchison, K. A. (2011). The interactive effects of listwide control, itembased control, and working memory capacity on Stroop performance. Journal of Experimental Psychology: Learning, Memory, and Cognition, 37(4), 851-860. doi:10.1037/a0023437

Hutchison, K. A., Bugg, J. M., Lim, Y., \& Olsen, M. (in press). Congruency pre-cues moderate item-specific proportion congruent effects. Attention, Perception, \& Psychophysics.

Jacoby, L. L., Lindsay, D., \& Hessels, S. (2003). Item-specific control of automatic processes: Stroop process dissociations. Psychonomic Bulletin \& Review, 10(3), 638-644. doi:10.3758/BF03196526

Kane, M. J., \& Engle, R. W. (2003). Working-memory capacity and the control of attention: The contributions of goal neglect, response competition, and task set to Stroop interference. Journal of Experimental Psychology: General, 132(1), 47-70. doi:10.1037/ 0096-3445.132.1.47
Lindsay, D., \& Jacoby, L. L. (1994). Stroop process dissociations: The relationship between facilitation and interference. Journal of Experimental Psychology: Human Perception and Performance, 20(2), 219-234. doi:10.1037/0096-1523.20.2.219

Logan, G. D., \& Zbrodoff, N. (1979). When it helps to be misled: Facilitative effects of increasing the frequency of conflicting stimuli in a Stroop-like task. Memory \& Cognition, 7(3), 166-174. doi:10. 3758/BF03197535

Lowe, D. G., \& Mitterer, J. O. (1982). Selective and divided attention in a Stroop task. Canadian Journal of Psychology/Revue Canadienne De Psychologie, 36(4), 684-700. doi:10.1037/h0080661

MacLeod, C. M. (1991). Half a century of research on the Stroop effect: An integrative review. Psychological Bulletin, 109(2), 163-203. doi:10.1037/0033-2909.109.2.163

Miller, E. K., \& Cohen, J. D. (2001). An integrative theory of prefrontal cortex function. Annual Review of Neuroscience, 24, 167-202. doi: 10.1146/annurev.neuro.24.1.167

Paxton, J. L., Barch, D. M., Racine, C. A., \& Braver, T. S. (2008). Cognitive control, goal maintenance, and prefrontal function in healthy aging. Cerebral Cortex, 18(5), 1010-1028. doi:10.1093/ cercor/bhm 135

Schmidt, J. R. (2013). Temporal learning and list-level proportion congruency: Conflict adaptation or learning when to respond? PLOS ONE, 8, e0082320. doi:10.1371/journal.pone. 0082320

Schmidt, J. R. (2014). List-level transfer effects in temporal learning. Further complications for the list-level proportion congruent effect. Journal of Cognitive Psychology, 26, 373-385. doi:10.1080/ 20445911.2014.896367

Schmidt, J. R., \& Besner, D. (2008). The Stroop effect: Why proportion congruent has nothing to do with congruency and everything to do with contingency. Journal of Experimental Psychology: Learning Memory, and Cognition, 34(3), 514-523. doi:10.1037/0278-7393. 34.3.514

Schneider, W., Eschman, A., \& Zuccolotto, A. (2002). E-prime reference guide. Pittsburgh, PA: Psychology Software Tools.

Shedden, J. M., Milliken, B., Watter, S., \& Monteiro, S. (2013). Eventrelated potentials as brain correlates of item specific proportion congruent effects. Consciousness and Cognition, 22(4), 1442-1455. doi:10.1016/j.concog.2013.10.002

Stroop, J. R. (1935). Studies of interference in serial verbal reactions Journal of Experimental Psychology, 18(6), 643-662. doi:10. 1037/h0054651

Thomas, D., \& Zumbo, B. D. (2012). Difference scores from the point of view of reliability and repeated-measures ANOVA: In defense of difference scores for data analysis. Educational and Psychological Measurement, 72(1), 37-43. doi:10.1177/0013164411409929

Tzelgov, J., Henik, A., \& Berger, J. (1992). Controlling Stroop effects by manipulating expectations for color words. Memory \& Cognition, 20(6), 727-735. doi:10.3758/BF03202722

Verguts, T., \& Notebaert, W. (2008). Hebbian learning of cognitive control: Dealing with specific and nonspecific adaptation. Psychological Review, 115(2), 518-525. doi:10.1037/0033-295X. 115.2 .518 